\title{
Fosfomycin and Comparator Activity Against Select Enterobacteriaceae, Pseudomonas, and Enterococcus Urinary Tract Infection Isolates from the United States in 2012
}

Tiffany R. Keepers · Marcela Gomez • Chris Celeri •

Kevin M. Krause · Donald Biek · Ian Critchley

Received: January 24, 2017 / Published online: March 11, 2017

(C) The Author(s) 2017. This article is published with open access at Springerlink.com

\section{ABSTRACT}

Introduction: Fosfomycin is a broad-spectrum cell wall active agent that inhibits the MurA enzyme involved in peptidoglycan synthesis and is FDA-approved for treatment of uncomplicated urinary tract infections (UTIs) caused by Escherichia coli and Enterococcus faecalis in women. Data regarding the susceptibility of recent UTI isolates to fosfomycin are limited.

Methods: This study compared the fosfomycin susceptibility of 658 US UTI isolates with susceptibility to ciprofloxacin, levofloxacin, nitrofurantoin, and trimethoprim/sulfamethoxazole (SXT). Isolates included E. coli $(n=257), K l e b-$ siella spp. $(n=156)$, Enterobacter spp. $(n=79)$, Pseudomonas aeruginosa $(n=60), \quad E$. faecalis $(n=54), \quad$ and Proteus spp. $(n=52)$. Extended-spectrum $\beta$-lactamase (ESBL)-producing E. coli, Klebsiella spp., and Proteus mirabilis,

Enhanced content To view enhanced content for this article go to http://www.medengine.com/Redeem/ 04F7F06050FD1A20.

T. R. Keepers · M. Gomez · C. Celeri ·

K. M. Krause · D. Biek - I. Critchley

Cerexa, Inc., 2100 Franklin Street \#900, Oakland,

CA 94612, USA

Present Address:

I. Critchley $(\square)$

Allergan Plc, 2525 Dupont Drive, Irvine, CA 92612, USA

e-mail: critchley_ian@allergan.com ceftazidime-nonsusceptible $P$. aeruginosa and Enterobacter spp., and vancomycin-nonsusceptible $E$. faecalis were included.

Results: Overall, the minimum concentration inhibiting 50\% of isolates $\left(\mathrm{MIC}_{50}\right)$ and $90 \%$ of isolates $\left(\mathrm{MIC}_{90}\right)$ for fosfomycin were 4 and $64 \mu \mathrm{g} / \mathrm{mL}$, respectively. Of the 257 E. coli isolates, $99.6 \%$ were susceptible to fosfomycin. Ciprofloxacin, levofloxacin, SXT, and nitrofurantoin susceptibility rates were $65.4 \%, 65.8 \%$, $59.9 \%$, and $90.3 \%$, respectively. The fosfomycin-susceptibility rate for $E$. faecalis $(94.4 \%)$ was comparable with the nitrofurantoin-susceptibility rate (98.1\%). Among the 144 ESBL-producing isolates, the fosfomycin $\mathrm{MIC}_{50}$ and $\mathrm{MIC}_{90}$ values were 2 and $32 \mu \mathrm{g} / \mathrm{mL}$, respectively. Fosfomycin $\mathrm{MIC}_{50}$ and $\mathrm{MIC}_{90}$ values were 16 and $128 \mu \mathrm{g} / \mathrm{mL}$ for the 38 ceftazidime-nonsusceptible Enterobacter isolates and 64 and $128 \mu \mathrm{g} / \mathrm{mL}$ for the 15 ceftazidime-nonsusceptible $P$. aeruginosa isolates, respectively.

Conclusion: These results demonstrate that fosfomycin has in vitro activity against many US UTI isolates, including drug-resistant isolates, and may provide another therapeutic option for treatment of UTIs caused by antibiotic-resistant pathogens.

Keywords: Antimicrobial; Fosfomycin; Urinary tract infection 


\section{INTRODUCTION}

Uncomplicated urinary tract infections (UTIs) are one of the most common infections for which antibiotics are prescribed, and there is a current trend of increasing resistance to antibiotics typically used to treat these infections [1]. Contributing to this increased resistance is the increased frequency of extended spectrum $\beta$-lactamase (ESBL) production in organisms causing UTIs, particularly Escherichia coli and Klebsiella spp [2, 3].

Fosfomycin, trimethoprim/sulfamethoxazole (SXT), and nitrofurantoin are the 3 treatment options recommended for uncomplicated UTIs by the Infectious Diseases Society of America (IDSA) [1]. To safeguard against development of resistance, fluoroquinolones, such as ciprofloxacin and levofloxacin, are not recommended for use in treatment of uncomplicated UTI, despite their effectiveness. The IDSA guidelines also recommend that ampicillin not be used because of the increased prevalence of ESBL-producing organisms in uncomplicated UTIs, and that $\beta$-lactam antibiotics are used only when other treatment options are unavailable [1].

Ciprofloxacin and SXT resistance rates among urine isolates from female outpatients increased dramatically from 2003 to 2012, especially among elderly outpatients, highlighting the need for other oral therapeutic alternatives [4]. In a study of 1518 extraintestinal E. coli isolates, including 1034 urinary isolates, recovered from patients in the United States and Germany between 2010 and 2011, investigators observed resistance rates to nitrofurantoin, ciprofloxacin, and SXT of $6.8 \%$, $17.1 \%$, and $26.9 \%$, respectively [5].

Fosfomycin is a bactericidal, cell wall active agent that binds to the MurA enzyme, thereby inhibiting the first step in peptidoglycan formation [6, 7]. It is approved, as fosfomycin tromethamine for oral administration, by the US Food and Drug Administration (FDA) for the treatment of women with uncomplicated UTIs (acute cystitis) caused by E. coli and Enterococcus faecalis and has broad-spectrum activity in vitro against Gram-positive and Gram-negative bacteria [8]. Fosfomycin has recently received renewed interest because of its activity against multidrug-resistant bacteria $[7,9,10]$ and the low frequency of cross-resistance to it [8], likely due to its unique mechanism of action. Furthermore, fosfomycin has low and stable resistance rates worldwide (1-3\% of E. coli) despite more than 20 years of clinical use in some countries $[11,12]$. In cases where fosfomycin resistance does emerge, common mechanisms include mutations affecting transport proteins (e.g., GlpT and UhpT) and mutation of its target, MurA [11].

This study sought to compare the sensitivity of UTI isolates to fosfomycin with sensitivity to antibiotics commonly used for treatment of UTIs, including ciprofloxacin, levofloxacin, nitrofurantoin, and SXT, among US isolates from the 2012 Assessing Worldwide Antimicrobial Resistance Evaluation (AWARE) surveillance program. AWARE is a global surveillance program established to monitor the susceptibility of prespecified pathogens to a number of antimicrobial agents [13]. Medical centers from the nine US census regions contributed to the database, with organisms collected being sent for susceptibility testing to a central laboratory (JMI Laboratories, North Liberty, IA, USA) [13]. Isolates for the present study were selected proportionally to reflect the most commonly isolated species from uncomplicated UTIs.

To test the hypothesis that fosfomycin has activity against typical urinary tract pathogens, the activity against isolates with a resistant phenotype, ESBL-positive E. coli, Klebsiella spp., and Proteus spp. was assessed, as was the activity of fosfomycin against ceftazidime-nonsusceptible $P$. aeruginosa, Enterobacter spp., and vancomycin-nonsusceptible E. faecalis isolates.

\section{METHODS}

\section{Isolates and Reagents}

Isolates collected during the 2012 AWARE surveillance program were retrospectively obtained from JMI Laboratories. Isolates were 
selected to obtain similar numbers of each species from each of the nine US census regions, based on their regional prevalence in the AWARE program [14]. Isolates $(n=658)$ included $E$. coli $(n=257)$, Klebsiella spp. $(n=156)$, Enterobacter spp. $(n=79), P$. aeruginosa $(n=60), E$. faecalis $(n=54)$, and Proteus spp. $(n=52)$. Resistant isolates included E. coli, Klebsiella spp., and P. mirabilis with an ESBL phenotype; ceftazidime-nonsusceptible $P$. aeruginosa and Enterobacter spp.; and vancomycin-nonsusceptible $E$. faecalis. The ESBL-phenotype was previously determined by JMI Laboratories and defined according to the Clinical and Laboratory Standards Institute (CLSI) ESBL screening criteria (ceftazidime and/or ceftriaxone and/or aztreonam minimum inhibitory concentration [MIC] value $>1 \mu \mathrm{g} / \mathrm{mL}$ ) [15]. Ceftazidime non-susceptibility (MICs $\geq 8 \mu \mathrm{g} / \mathrm{mL}$ ) was used to categorize Enterobacter spp. Ceftriaxone and vancomycin susceptibilities were determined by JMI Laboratories using CLSI breakpoints [15]. Genotype determinations were made by JMI Laboratories using Check-points microarray kit CT-101 (Check-Points, Wageningen, The Netherlands) $[16,17]$. This kit has the capability to detect bla $_{\text {CTX-M }}$ Groups 1, 2, $8+25$, and $9, b l a_{\text {TEM }}$ wild-type (wt) and ESBL, $b l a_{\mathrm{SHV}}$ wt and ESBL, $b l a_{\mathrm{ACC}}, b l a_{\mathrm{ACT} / \mathrm{MIR}}, b l a_{\mathrm{C}-}$ MYII $b l a_{\mathrm{DHA}}, b l a_{\mathrm{FOX}}, b l a_{\mathrm{KPC}}$, and $b l a_{\mathrm{NDM}-1}$ (but not $b l a_{\mathrm{OXA}-48}, b l a_{\mathrm{IMP}}$ or $\left.b l a_{\mathrm{VIM}}\right)$.

Antibiotics included in this study were fosfomycin (P5396-5G, lot BCBJ9873V; Sigma-Aldrich, St. Louis, MO, USA), ciprofloxacin (1134335, lot J0H307; USP, Rockville, MD, USA), levofloxacin (28266-1G-F, lot 1425507; Sigma-Aldrich, St. Louis, MO, USA), nitrofurantoin (1464001, lot K01060; USP), trimethoprim (Sigma T7883-5G, lot BCBB6418; Sigma-Aldrich), and sulfamethoxazole (Sigma S7507-10G, lot 048K0124; Sigma-Aldrich). Reagents used in this study included Mueller-Hinton agar (MHA; BBL 211438, lot 3217394; BD Diagnostic Systems, Sparks, MD, USA), glucose-6-phosphate (G7879-1G, lot 031M7004V; Sigma-Aldrich), and cation-adjusted Mueller-Hinton broth (CAMHB; BBL 21322; BD Diagnostic Systems).

\section{Agar Dilution}

Minimum inhibitory concentration for fosfomycin was determined by agar dilution according to CLSI documents M07-A9 and M100-S24 [15, 18]. MHA was prepared according to manufacturer's instructions followed by the addition of glucose-6-phosphate to a final concentration of $25 \mu \mathrm{g} / \mathrm{mL}$. Fosfomycin was added to MHA in 2-fold serial dilutions at concentrations ranging from 0.25 to $256 \mu \mathrm{g} / \mathrm{mL}$. Approximately $10^{4}$ colony-forming units (CFU)/ spot were added to plates. After incubation at $35 \pm 2{ }^{\circ} \mathrm{C}$ for $16-20 \mathrm{~h}$, the MIC was recorded as the lowest concentration of antimicrobial agent that completely inhibited visible growth.

\section{Broth Microdilution}

Minimum inhibitory concentration values for ciprofloxacin, levofloxacin, nitrofurantoin, and SXT were determined by broth microdilution methods according to CLSI documents M07-A9 and M100-S24 [15, 18], with testing of appropriate quality control strains.

Antibiotic test plates were prepared in-house using a twofold dilution series in 96-well round-bottom plates containing antibiotic $(10 \times$ solution). Bacterial suspensions were diluted in CAMHB and added to the antibiotic test plates to yield a final culture density of $5 \times 10^{5} \mathrm{CFU} /$ $\mathrm{mL}$. After incubation for $18 \mathrm{~h}$ at $35 \pm 2{ }^{\circ} \mathrm{C}$, the MIC was recorded as the lowest concentration where there was no visible bacterial growth.

\section{Analysis}

Minimum inhibitory concentration values were interpreted with respect to CLSI interpretative criteria for $E$. coli and $E$. faecalis [fosfomycin breakpoints were MIC $\leq 64 \mu \mathrm{g} / \mathrm{mL}$ (susceptible), $\mathrm{MIC}=128 \mu \mathrm{g} / \mathrm{mL} \quad$ (intermediate), and MIC $\geq 256 \mu \mathrm{g} / \mathrm{mL}$ (resistant)] [15].

\section{Compliance with Ethics Guidelines}

All procedures followed were in accordance with the ethical standards of the responsible 
committee on human experimentation (institutional and national) and with the Helsinki Declaration of 1964, as revised in 2008. Informed consent was not required because isolates used in this study were from a previously conducted surveillance program and no patient-identifiable data were held by the investigators undertaking the current research.

\section{RESULTS}

A total of 658 UTI isolates were tested from the nine US census regions representing defined numbers of isolates of selected species collected during the 2012 AWARE surveillance program. Overall, MIC values for fosfomycin ranged from $\leq 0.25$ to $>256 \mu \mathrm{g} / \mathrm{mL}$, and the fosfomycin $\mathrm{MIC}_{50}$ and $\mathrm{MIC}_{90}$ values for all isolates were 4 and $64 \mu \mathrm{g} / \mathrm{mL}$, respectively (Table 1). A total of 202 isolates had a resistant phenotype (Table 2). Overall, 21.9\% (144/658) of isolates were of the ESBL-phenotype (E. coli, $K$. pneumoniae, K. oxytoca, and P. mirabilis only), $53(8.1 \%)$ were ceftazidime-nonsusceptible $E$. cloacae and $P$. aeruginosa isolates and 5 $(0.8 \%)$ were vancomycin-nonsusceptible E. faecalis isolates.

Susceptibility results of the $257 \mathrm{E}$. coli isolates tested are shown in Table 1 . The fosfomycin $\mathrm{MIC}_{50}$ and $\mathrm{MIC}_{90}$ values for E. coli were 1 and $4 \mu \mathrm{g} / \mathrm{mL}$, respectively. Only one (0.4\%) E. coli isolate (an ESBL-phenotype) was resistant to fosfomycin. Susceptibility results of the 76 E. coli isolates with an ESBL phenotype are shown in Table 2.

Summaries of pathogen susceptibilities are provided in Tables 1 and 2. Fosfomycin had $\mathrm{MIC}_{50}$ and $\mathrm{MIC}_{90}$ values of 8 and $32 \mu \mathrm{g} / \mathrm{mL}$ for Klebsiella spp., 4 and $32 \mu \mathrm{g} / \mathrm{mL}$ for Proteus spp., and 16 and $32 \mu \mathrm{g} / \mathrm{mL}$ for Enterobacter spp., respectively (Table 1$)$. Most E. faecalis isolates $(94.4 \%)$ were susceptible to fosfomycin (Table 1). Fosfomycin $\mathrm{MIC}_{50}$ and $\mathrm{MIC}_{90}$ values against $P$. aeruginosa were 64 and $128 \mu \mathrm{g} / \mathrm{mL}$, respectively (Table 1). Among all 202 resistant organisms tested (including those with an ESBL phenotype, ceftazidime-nonsusceptible Gram-negative pathogens, and vancomycin-nonsusceptible $E$. faecalis), fosfomycin had $\mathrm{MIC}_{50}$ and $\mathrm{MIC}_{90}$ values of 4 and $64 \mu \mathrm{g} / \mathrm{mL}$, respectively (Table 2 ). Twenty-four of these isolates $(23 \mathrm{~K}$. pneumoniae and $1 \mathrm{E}$. coli) were carbapenemase producers based on the detection of a KPC gene and on being nonsusceptible to meropenem (data not shown). All but two of these carbapenem resistant isolates had MICs indicative of susceptibility to fosfomycin (based on the CLSI interpretive guidelines for $E$. coli and E. faecalis, one was intermediate and one resistant). There were only 5 vancomycin-nonsusceptible $E$. faecalis tested, but all were susceptible to fosfomycin with MIC values $\leq 64 \mu \mathrm{g} /$ $\mathrm{mL}$ (Table 2). Fosfomycin $\mathrm{MIC}_{50}$ and $\mathrm{MIC}_{90}$ values for Klebsiella spp. with an EBSL-phenotype were 8 and $128 \mu \mathrm{g} / \mathrm{mL}$ and for ceftazidime-nonsusceptible Enterobacter spp. were 16 and $128 \mu \mathrm{g} / \mathrm{mL}$, respectively (Table 2 ). By comparison, the fosfomycin $\mathrm{MIC}_{50}$ for ceftazidime-nonsusceptible $P$. aeruginosa was numerically higher $(64 \mu \mathrm{g} / \mathrm{mL})$, whereas the $\mathrm{MIC}_{90}$ value was similar $(128 \mu \mathrm{g} / \mathrm{mL}$; Table 2).

In total, $17(2.6 \%)$ isolates had MIC values suggesting resistance to fosfomycin (data not shown), including E. coli (ESBL-phenotype; $n=1)$, ceftazidime-nonsusceptible $E$. cloacae $(n=3)$, vancomycin-susceptible $E$. faecalis $(n=1), K$. pneumoniae $[n=10$; ESBL-phenotype $(n=5)$ and non-ESBL-phenotype $(n=5)]$, non-ESBL-phenotype $P$. mirabilis $(n=1)$, and ceftazidime-nonsusceptible $\quad P$. aeruginosa $(n=1)$. Among those isolates with an ESBL-phenotype that were fosfomycin-"resis$\operatorname{tant} "(n=6)$, there was no common $\beta$-lactamase gene detected among all the isolates, and there was no clear correlation between $\beta$-lactamase genes present and fosfomycin resistance (data not shown). Three isolates with $b l a_{\text {СтХ-м }}$ genes were resistant to fosfomycin $(2 \mathrm{~K}$. pneumoniae and 1 E. coli).

\section{DISCUSSION}

Overall, fosfomycin exhibited antibacterial activity against the panel of 658 organisms. With the exception of 1 isolate, all E. coli isolates tested were susceptible to fosfomycin. By comparison, the $E$. coli susceptibility rates were markedly lower for the other antibiotics tested, 
Table 1 Pathogen characteristics and antibiotic susceptibilities

\begin{tabular}{|c|c|c|c|c|c|c|}
\hline & $\mathrm{MIC}_{50}$ & $\mathrm{MIC}_{90}$ & Range & $S(\%)$ & $I(\%)$ & $R(\%)$ \\
\hline \multicolumn{7}{|c|}{ All organisms $(n=658)$} \\
\hline Fosfomycin & 4 & 64 & $\leq 0.25$ to $>256$ & - & - & - \\
\hline Ciprofloxacin & 0.06 & $>32$ & 0.004 to $>32$ & 68.4 & 3.0 & 28.6 \\
\hline Levofloxacin & 0.25 & 32 & 0.016 to $>32$ & 70.4 & 1.7 & 28.0 \\
\hline Nitrofurantoin ${ }^{\mathrm{a}}$ & 32 & 128 & 1 to $>128$ & 56.4 & 23.3 & 20.3 \\
\hline $\mathrm{SXT}^{\mathrm{b}}$ & 0.5 & $>32$ & 0.06 to $>32$ & 63.4 & - & 36.6 \\
\hline \multicolumn{7}{|l|}{ E. $\operatorname{coli}(n=257)$} \\
\hline Fosfomycin & 1 & 4 & $\leq 0.25$ to $>256$ & 99.6 & 0.0 & 0.4 \\
\hline Ciprofloxacin & 0.03 & $>32$ & 0.004 to $>32$ & 65.4 & 0.0 & 34.6 \\
\hline Levofloxacin & 0.06 & 32 & 0.016 to $>32$ & 65.8 & 0.4 & 33.9 \\
\hline Nitrofurantoin & 16 & 32 & 2 to $>128$ & 90.3 & 5.8 & 3.9 \\
\hline SXT & 0.5 & $>32$ & 0.06 to $>32$ & 59.9 & - & 40.1 \\
\hline \multicolumn{7}{|c|}{ Klebsiella spp. $(n=156)^{\mathrm{c}}$} \\
\hline Fosfomycin & 8 & 32 & 0.5 to $>256$ & - & - & - \\
\hline Ciprofloxacin & 0.06 & $>32$ & 0.008 to $>32$ & 66.0 & 1.9 & 32.1 \\
\hline Levofloxacin & 0.125 & $>32$ & 0.016 to $>32$ & 68.6 & 1.3 & 30.1 \\
\hline Nitrofurantoin & 64 & 128 & 2 to 128 & 9.0 & 41.0 & 50.0 \\
\hline SXT & 0.5 & $>32$ & 0.06 to $>32$ & 58.3 & - & 41.7 \\
\hline \multicolumn{7}{|c|}{ Proteus spp. $(n=52)^{\mathrm{d}}$} \\
\hline Fosfomycin & 4 & 32 & $\leq 0.25$ to $>256$ & - & - & - \\
\hline Ciprofloxacin & 0.03 & 4 & 0.016 to $>32$ & 86.5 & 1.9 & 11.5 \\
\hline Levofloxacin & 0.06 & 4 & $\leq 0.03$ to 32 & 88.5 & 3.8 & 7.7 \\
\hline Nitrofurantoin & - & - & - & - & - & - \\
\hline SXT & 0.25 & $>32$ & 0.06 to $>32$ & 73.1 & - & 26.9 \\
\hline \multicolumn{7}{|c|}{ Enterobacter spp. $(n=79)^{\mathrm{e}}$} \\
\hline Fosfomycin & 16 & 32 & 0.5 to $>256$ & - & - & - \\
\hline Ciprofloxacin & 0.03 & 32 & 0.008 to $>32$ & 83.5 & 5.1 & 11.4 \\
\hline Levofloxacin & 0.06 & 32 & 0.016 to $>32$ & 83.5 & 5.1 & 11.4 \\
\hline Nitrofurantoin & 64 & 128 & 1 to 128 & 11.4 & 59.5 & 29.1 \\
\hline SXT & 0.5 & $>32$ & 0.06 to $>32$ & 78.5 & - & 21.5 \\
\hline \multicolumn{7}{|c|}{ CAZ S Enterobacter spp. $(n=41)^{\mathrm{f}}$} \\
\hline Fosfomycin & 16 & 32 & 0.5 to 128 & - & - & - \\
\hline Ciprofloxacin & 0.03 & 0.25 & 0.016 to 32 & 97.6 & 0.0 & 2.4 \\
\hline Levofloxacin & 0.06 & 0.25 & 0.016 to 32 & 97.6 & 0.0 & 2.4 \\
\hline Nitrofurantoin & 64 & 128 & 2 to 128 & 7.3 & 58.5 & 34.1 \\
\hline SXT & 0.25 & 2 & $\leq 0.25$ to $>32$ & 90.2 & - & 9.8 \\
\hline \multicolumn{7}{|l|}{ E. faecalis $(n=54)$} \\
\hline Fosfomycin & 32 & 64 & 16 to $>256$ & 94.4 & 3.7 & 1.9 \\
\hline Ciprofloxacin & 1 & $>32$ & 0.5 to $>32$ & 51.9 & 16.7 & 31.5 \\
\hline Levofloxacin & 1 & $>32$ & 0.5 to $>32$ & 68.5 & 0.0 & 31.5 \\
\hline Nitrofurantoin & 8 & 16 & 8 to 64 & 98.1 & 1.9 & 0.0 \\
\hline SXT & - & - & - & - & - & - \\
\hline \multicolumn{7}{|c|}{ VAN S E. faecalis $(n=49)$} \\
\hline Fosfomycin & 64 & 64 & 16 to 256 & 93.9 & 4.1 & 2.0 \\
\hline Ciprofloxacin & 1 & $>32$ & 0.5 to $>32$ & 57.1 & 18.4 & 24.5 \\
\hline
\end{tabular}


Table 1 continued

\begin{tabular}{|c|c|c|c|c|c|c|}
\hline & $\mathrm{MIC}_{50}$ & $\mathrm{MIC}_{90}$ & Range & $S(\%)$ & $I(\%)$ & $\overline{R(\%)}$ \\
\hline Levofloxacin & 1 & $>32$ & 0.5 to $>32$ & 75.5 & 0.0 & 24.5 \\
\hline Nitrofurantoin & 8 & 16 & 8 to 64 & 98.0 & 2.0 & 0.0 \\
\hline SXT & - & - & - & - & - & - \\
\hline \multicolumn{7}{|c|}{ P. aeruginosa $(n=60)$} \\
\hline Fosfomycin & 64 & 128 & 2 to $>256$ & - & - & - \\
\hline Ciprofloxacin & 0.25 & 32 & 0.008 to $>32$ & 66.7 & 5.0 & 28.3 \\
\hline Levofloxacin & 1 & $>32$ & 0.016 to $>32$ & 63.3 & 3.3 & 33.3 \\
\hline Nitrofurantoin & - & - & - & - & - & - \\
\hline SXT & - & - & - & - & - & - \\
\hline \multicolumn{7}{|c|}{ CAZ S $P$. aeruginosa $(n=45)$} \\
\hline Fosfomycin & 64 & 128 & 4 to 128 & - & - & - \\
\hline Ciprofloxacin & 0.125 & 32 & 0.008 to $>32$ & 84.4 & 0.0 & 15.6 \\
\hline Levofloxacin & 0.5 & $>32$ & 0.016 to $>32$ & 80.0 & 4.4 & 15.6 \\
\hline Nitrofurantoin & - & - & - & - & - & - \\
\hline SXT & - & - & - & - & - & - \\
\hline
\end{tabular}

Percent susceptible not calculable due to organisms in the group without CLSI breakpoints indicated by " - "

$C A Z S$ ceftazidime-susceptible, $I$ intermediate, $M I C_{50}$ minimum concentration that inhibits $50 \%$ of isolates, $M I C_{90}$ minimum concentration that inhibits $90 \%$ of isolates, $R$ resistant, $S$ susceptible, $S X T$ trimethoprim/sulfamethoxazole, $V A N S$ vancomycin-susceptible

${ }^{a} P$. aeruginosa and Proteus spp. $(n=546)$ are intrinsically resistant to nitrofurantoin and were not included in the calculations

b $P$. aeruginosa and E. faecalis $(n=544)$ are intrinsically resistant to SXT, so those organisms were not included in the calculations

' $K$. pneumoniae $(n=36) ;$. oxytoca $(n=20)$

d P. mirabilis $(n=34) ;$ P. vulgaris $(n=18)$

' E. cloacae $(n=49) ; E$. aerogenes $(n=30)$

${ }^{\mathrm{f}}$ E. cloacae $(n=26) ;$ E. aerogenes $(n=15)$

a significant finding given that $E$. coli is the pathogen most commonly found in UTI infections. The fosfomycin susceptibility rate for E. coli isolates with an ESBL-phenotype was markedly higher than any other antibiotic tested. Among the isolates with an ESBL phenotype, $b l a_{\mathrm{CTX}-\mathrm{M}}$ group 1 and non-ESBL $b l a_{\mathrm{TEM}}$ were the most common $\beta$-lactamase genes detected (data not shown).

A study of E. coli isolated from urine of US outpatients from 2000 to 2010 reported resistance rates for ciprofloxacin, nitrofurantoin, and SXT that were slightly lower than those observed in the present study; this likely reflects a continued increase in resistance over the period 2010 to 2012 similar to that observed for the period 2000 to 2010 [20]. A recent study of E. coli isolates from patients with UTI in Canada during the period from 2010 to 2013 demonstrated susceptibility rates similar to the present study for fosfomycin $(99.4 \%$ vs. $99.6 \%$, respectively) and nitrofurantoin (96.1\% vs. $90.3 \%$, respectively) and higher than observed for ciprofloxacin $(77.4 \%$ vs. $65.4 \%$, respectively), and SXT (74.7\% vs. $59.9 \%$, respectively) [21]. US researchers also found $E$. coli to commonly exhibit susceptibility to fosfomycin (100\%, tested using agar dilution) and nitrofurantoin (98.0\%) [19].

Compared with the present study, similar susceptibility rates were also reported among ESBL-producing $E$. coli isolates from patients with UTI in the Canadian study for fosfomycin $(100 \%)$ and nitrofurantoin $(83.3 \%)$, with SXT $(35.7 \%)$, and ciprofloxacin $(9.5 \%)$ rates being somewhat lower [21]. Similarly, in a small US study, ESBL-producing E. coli isolates were susceptible to fosfomycin (96.7\%; MIC values ranged from 0.25 to $4 \mu \mathrm{g} / \mathrm{mL})$, but less so to nitrofurantoin (76.7\%; MIC values of $8-64 \mu \mathrm{g} /$ $\mathrm{mL}$; susceptibility breakpoint $\leq 32 \mu \mathrm{g} / \mathrm{mL}$ ) [22]. Another study of $E$. coli isolated from patients with UTI in China from 2004 to 2012 showed that fosfomycin susceptibility rates were high 
Table 2 Pathogen characteristics and antibiotic susceptibilities for resistant isolates

\begin{tabular}{|c|c|c|c|c|c|c|}
\hline & $\mathrm{MIC}_{50}$ & $\mathrm{MIC}_{90}$ & Range & $S(\%)$ & $I(\%)$ & $R(\%)$ \\
\hline \multicolumn{7}{|c|}{ Resistant organisms $(n=202)$} \\
\hline Fosfomycin & 4 & 64 & $\leq 0.25$ to $>256$ & - & - & - \\
\hline Ciprofloxacin & 32 & $>32$ & 0.016 to $>32$ & 32.2 & 5.0 & 62.9 \\
\hline Levofloxacin & 16 & $>32$ & 0.016 to $>32$ & 33.7 & 3.0 & 63.4 \\
\hline Nitrofurantoin $^{\mathrm{a}}$ & 64 & 128 & 1 to 128 & 41.8 & 25.5 & 32.6 \\
\hline $\mathrm{SXT}^{\mathrm{b}}$ & $>32$ & $>32$ & 0.06 to $>32$ & 40.7 & - & 59.3 \\
\hline \multicolumn{7}{|c|}{ ESBL-phenotype $(n=144)^{\mathrm{c}}$} \\
\hline Fosfomycin & 2 & 32 & $\leq 0.25$ to $>256$ & - & - & - \\
\hline Ciprofloxacin & $>32$ & $>32$ & 0.016 to $>32$ & 25.7 & 2.1 & 72.2 \\
\hline Levofloxacin & 16 & $>32$ & 0.016 to $>32$ & 27.8 & 1.4 & 70.8 \\
\hline Nitrofurantoin ${ }^{\mathrm{d}}$ & 64 & 128 & 8 to 128 & 46.8 & 17.0 & 36.2 \\
\hline SXT & $>32$ & $>32$ & 0.06 to $>32$ & 34.0 & - & 66.0 \\
\hline \multicolumn{7}{|c|}{ E. coli ESBL-phenotype $(n=76)$} \\
\hline Fosfomycin & 1 & 4 & $\leq 0.25$ to $>256$ & 98.7 & 0.0 & 1.3 \\
\hline Ciprofloxacin & $>32$ & $>32$ & 0.016 to $>32$ & 28.9 & 0.0 & 71.1 \\
\hline Levofloxacin & 16 & 32 & 0.03 to $>32$ & 28.9 & 1.3 & 69.7 \\
\hline Nitrofurantoin & 16 & 64 & 8 to 128 & 82.9 & 9.2 & 7.9 \\
\hline SXT & $>32$ & $>32$ & 0.06 to $>32$ & 43.4 & - & 56.6 \\
\hline \multicolumn{7}{|c|}{ Klebsiella spp.: ESBL-phenotype $(n=65)^{\mathrm{e}}$} \\
\hline Fosfomycin & 8 & 128 & 1 to $>256$ & - & - & - \\
\hline Ciprofloxacin & 32 & $>32$ & 0.016 to $>32$ & 21.5 & 4.6 & 73.8 \\
\hline Levofloxacin & 16 & $>32$ & 0.016 to $>32$ & 26.2 & 1.5 & 72.3 \\
\hline Nitrofurantoin & 128 & 128 & 16 to $>256$ & 4.6 & 26.2 & 69.2 \\
\hline SXT & $>32$ & $>32$ & 0.06 to $>32$ & 23.1 & - & 76.9 \\
\hline \multicolumn{7}{|c|}{ P. mirabilis: ESBL-phenotype $(n=3)$} \\
\hline Fosfomycin & $\mathrm{NC}$ & $\mathrm{NC}$ & 0.5 to 2 & - & - & - \\
\hline Ciprofloxacin & $\mathrm{NC}$ & $\mathrm{NC}$ & 0.03 to $>32$ & 33.3 & 0.0 & 66.7 \\
\hline Levofloxacin & $\mathrm{NC}$ & $\mathrm{NC}$ & 0.125 to 32 & 33.3 & 0.0 & 66.7 \\
\hline Nitrofurantoin & - & - & - & 0.0 & 33.3 & 66.7 \\
\hline SXT & $\mathrm{NC}$ & $\mathrm{NC}$ & 1 to $>32$ & 33.3 & - & 66.7 \\
\hline \multicolumn{7}{|c|}{ CAZ NS Enterobacter spp. $(n=38)^{\mathrm{f}}$} \\
\hline Fosfomycin & 16 & 128 & 0.5 to $>256$ & - & - & - \\
\hline Ciprofloxacin & 0.25 & $>32$ & 0.008 to $>32$ & 68.4 & 10.5 & 21.1 \\
\hline
\end{tabular}


Table 2 continued

\begin{tabular}{|c|c|c|c|c|c|c|}
\hline & $\mathrm{MIC}_{50}$ & $\mathrm{MIC}_{90}$ & Range & $S(\%)$ & $I(\%)$ & $R(\%)$ \\
\hline Levofloxacin & 0.25 & 32 & 0.03 to $>32$ & 68.4 & 10.5 & 21.1 \\
\hline Nitrofurantoin & 64 & 128 & 1 to 128 & 15.8 & 60.5 & 23.7 \\
\hline SXT & $0.5 / 9.5$ & $>32 / 608$ & 0.06 to $>32$ & 65.8 & - & 34.2 \\
\hline \multicolumn{7}{|c|}{ CAZ NS $P$. aeruginosa $(n=15)$} \\
\hline Fosfomycin & 64 & 128 & 2 to $>256$ & - & - & - \\
\hline Ciprofloxacin & 16 & 32 & 0.125 to 32 & 13.3 & 20.0 & 66.7 \\
\hline Levofloxacin & 16 & $>32$ & 0.5 to $>32$ & 13.3 & 0.0 & 86.7 \\
\hline Nitrofurantoin & - & - & - & - & - & - \\
\hline SXT & - & - & - & - & - & - \\
\hline \multicolumn{7}{|c|}{ VAN NS $E$. faecalis $(n=5)$} \\
\hline Fosfomycin & $\mathrm{NC}$ & $\mathrm{NC}$ & 32 to 64 & 100.0 & 0.0 & 0.0 \\
\hline Ciprofloxacin & $\mathrm{NC}$ & $\mathrm{NC}$ & $>32$ to $>32$ & 0.0 & 0.0 & 100.0 \\
\hline Levofloxacin & $\mathrm{NC}$ & $\mathrm{NC}$ & 32 to $>32$ & 0.0 & 0.0 & 100.0 \\
\hline Nitrofurantoin & $\mathrm{NC}$ & $\mathrm{NC}$ & 8 to 16 & 100.0 & 0.0 & 0.0 \\
\hline SXT & - & - & - & - & - & - \\
\hline
\end{tabular}

Percent susceptible not calculable due to organisms in the group without CLSI breakpoints indicated by "-”

$C A Z N S$ ceftazidime-nonsusceptible, $E S B L$ extended spectrum $\beta$-lactamase, $I$ intermediate, $M I C_{50}$ minimum concentration that inhibits $50 \%$ of isolates, $M I C_{90}$ minimum concentration that inhibits $90 \%$ of isolates, $R$ resistant, $S$ susceptible, $S X T$ trimethoprim/sulfamethoxazole, $V A N N S$ vancomycin-nonsusceptible

${ }^{a} P$. aeruginosa and Proteus spp. $(n=184)$ are intrinsically resistant to nitrofurantoin and were not included in the calculations

b $P$. aeruginosa and $E$. faecalis $(n=182)$ are intrinsically resistant to SXT and were not included in the calculations

${ }^{c}$ E. coli $(n=76), K$. pneumoniae $(n=59)$, K. oxytoca $(n=6)$, and P. mirabilis $(n=3)$

d $P$. mirabilis $(n=141)$ are intrinsically resistant to nitrofurantoin and were not included in the calculations

e $K$. pneumoniae $(n=59)$ and $K$. oxytoca $(n=6)$

f E. cloacae $(n=23)$ and E. aerogenes $(n=15)$

(93.4-99.4\%), despite high ESBL-producing phenotype rates of $58.1 \%$ among isolates tested; ESBL-producing E. coli susceptibility rates for fosfomycin (93.8\%) and nitrofurantoin (86.2\%) were also similar to those reported here for isolates with ESBL phenotypes [23]. Over the period 2010 through to 2013, susceptibility rates of $E$. coli isolates were similarly high for fosfomycin $(95.8 \%)$ in multidrug-resistant urinary isolates from a Veterans Affairs population in Boston, MA, USA [24].

The reduced susceptibility of cUTI isolates Klebsiella and Enterobacter spp. to nitrofurantoin observed in this study was also observed in a study conducted in Canada in 2001 (susceptibility rates of $69.2 \%$ and $63.0 \%$, respectively) [25], and in a recent US study (susceptibility rates of $52.3 \%$ and $71.9 \%$, respectively) [19]. Similarly, in the Boston area Veterans Affairs population, $64.0 \%$ of Klebsiella spp. were susceptible to nitrofurantoin, but in contrast to the current study slightly more than half of the multidrug-resistant isolates were susceptible to fosfomycin [24]. Ongoing surveillance is needed to assess the activity of nitrofurantoin against contemporary isolates of these organisms. 
Overall, the rate of fosfomycin resistance was low in this study, as has been recently reported in general populations in the US (resistance rates of $1.5 \%$ and $3.3 \%)[19,22]$, but not in a predominantly male population from the Boston area (overall resistance rate of 21.6\%) [24]. There are several mechanisms by which bacteria become resistant to fosfomycin, including amino acid substitutions that modify the fosfomycin target MurA, mutations that affect fosfomycin transporters, and acquisition of genes that encode enzymes that inactivate fosfomycin [11]. Although fosfomycin resistance is not typically associated with $\beta$-lactamase production, one study demonstrated that the fosA3 gene, which encodes a fosfomycin-inactivating enzyme, can be associated with $b a_{\mathrm{CTX}-\mathrm{M}}$ genes in E. coli [26]. This may explain the fosfomycin resistance observed in a few of the isolates assessed in this study. Further testing is needed to determine whether these fosfomycin- resistant pathogens encode the fos $A 3$ gene.

This study shares the limitations inherent to in vitro trials, including the potential limited applicability to the clinical setting. In this case, our study is further limited by relying on a subset of data from the AWARE surveillance program. The data presented here represent a subset of surveillance results of US isolates from the 2012 AWARE surveillance program, and although the data have been stratified by region and by pathogen to reflect the original database, it is possible that the susceptibility patterns of the actual isolates included in our study may have differed from the underlying AWARE data. Furthermore, the isolates that we could assess were limited to those isolates collected through the AWARE surveillance program and duplicate MIC testing was not performed. Nonetheless, the AWARE surveillance program offers a useful dataset from which to assess susceptibility patterns at a point in time. The clinical applicability of our study may be further limited as susceptibility rates may change over time or differ in other regions of the world.

\section{CONCLUSION}

The data reported in this study demonstrate that, compared with the other antibiotics tested, fosfomycin has greater in vitro activity against US UTI isolates, including Enterobacteriaceae with ESBL-phenotypes and other drug-resistant isolates. The susceptibility data for US surveillance isolates in this study should serve as a baseline for benchmarking any future changes in the susceptibility of uropathogens to fosfomycin as it becomes more widely used for the treatment of infections caused by resistant pathogens.

\section{ACKNOWLEDGEMENTS}

Sponsorship and article processing charges for this study were funded by Cerexa, Inc. (Oakland, CA, USA), a subsidiary of Allergan plc. Cerexa, Inc., was involved in the design, collection, analysis, interpretation of data, and decision to present these results. All authors had full access to all of the data in this study and take complete responsibility for the integrity of the data and accuracy of the data analysis. We would like to thank JMI Laboratories, North Liberty, IA, USA, for providing the isolates and performing the ESBL characterization. Writing and editorial assistance were provided to the authors by Todd J. Waldron, PhD, and John E. Fincke, PhD, of Complete Healthcare Communications, LLC (Chadds Ford, PA, USA), a CHC Group company. Support for this assistance was funded by Allergan plc. All named authors meet the International Committee of Medical Journals (ICMJE) criteria for authorship for this manuscript, take responsibility for the integrity of the work as a whole, and have given final approval to the version to be published.

Disclosures. T. R. Keepers was an employee of Cerexa, Inc. at the time of study conduct and analysis, and may own stock or stock options. M. Gomez was an employee of Cerexa, Inc. at the time of study conduct and analysis, and may own stock or stock options. C. Celeri was an employee of Cerexa, Inc. at the time of study conduct and analysis, and may own stock or stock options. K. M. Krause was an employee of Cerexa, Inc. at the time of study conduct and analysis, and may own stock or stock options. 
D. Biek was an employee of Cerexa, Inc. at the time of study conduct and analysis, and may own stock or stock options. D. Biek's current affiliation: DBiek Consulting, 1765 Walnut Drive, Mountain View, CA 94040, USA. I. Critchley was an employee of Cerexa, Inc. at the time of study conduct and analysis, and may own stock or stock options. I. Critchley's current affiliation: Allergan Plc, 2525 Dupont Drive, Irvine, CA 92612, USA.

Compliance with Ethics Guidelines. All procedures followed were in accordance with the ethical standards of the responsible committee on human experimentation (institutional and national) and with the Helsinki Declaration of 1964, as revised in 2008. Informed consent was not required because all isolates used in this study were from a previously conducted surveillance program and no patient-identifiable data were held by the investigators undertaking the current research.

Data Availability. The datasets generated during and/or analyzed during the current study are available from the corresponding author on reasonable request.

Open Access. This article is distributed under the terms of the Creative Commons Attribution-NonCommercial 4.0 International License (http://creativecommons.org/licenses/ by-nc/4.0/), which permits any noncommercial use, distribution, and reproduction in any medium, provided you give appropriate credit to the original author(s) and the source, provide a link to the Creative Commons license, and indicate if changes were made.

\section{REFERENCES}

1. Gupta K, Hooton TM, Naber KG, et al. International clinical practice guidelines for the treatment of acute uncomplicated cystitis and pyelonephritis in women: a 2010 update by the Infectious Diseases Society of America and the European Society for Microbiology and Infectious Diseases. Clin Infect Dis. 2011;52:e103-20.
2. Sader HS, Flamm RK, Jones RN. Frequency of occurrence and antimicrobial susceptibility of Gram-negative bacteremia isolates in patients with urinary tract infection: results from United States and European hospitals (2009-2011). J Chemother. 2014;26:133-8.

3. Bonkat G, Muller G, Braissant $\mathrm{O}$, et al. Increasing prevalence of ciprofloxacin resistance in extended-spectrum-beta-lactamase-producing Escherichia coli urinary isolates. World J Urol. 2013;31:1427-32.

4. Sanchez GV, Babiker A, Master RN, et al. Antibiotic resistance among urinary isolates from female outpatients in the United States in 2003 and 2012. Antimicrob Agents Chemother. 2016;60:2680-3.

5. Tchesnokova V, Billig M, Chattopadhyay S, et al. Predictive diagnostics for Escherichia coli infections based on the clonal association of antimicrobial resistance and clinical outcome. J Clin Microbiol. 2013;51:2991-9.

6. Skarzynski T, Mistry A, Wonacott A, et al. Structure of UDP- $N$-acetylglucosamine enolpyruvyl transferase, an enzyme essential for the synthesis of bacterial peptidoglycan, complexed with substrate UDP- $N$-acetylglucosamine and the drug fosfomycin. Structure. 1996;4:1465-74.

7. Michalopoulos AS, Livaditis IG, Gougoutas V. The revival of fosfomycin. Int $\mathrm{J}$ Infect Dis. 2011;15:e732-9.

8. $\mathrm{MONUROL}^{\circledR}$ (fosfomycin tromethamine). Full prescribing information, Forest Laboratories, LLC, St. Louis, MO, 2014.

9. Reffert JL, Smith WJ. Fosfomycin for the treatment of resistant gram-negative bacterial infections. Insights from the Society of Infectious Diseases Pharmacists. Pharmacotherapy. 2014;34:845-57.

10. Dinh A, Salomon J, Bru JP, Bernard L. Fosfomycin: efficacy against infections caused by multidrug-resistant bacteria. Scand J Infect Dis. 2012;44:182-9.

11. Karageorgopoulos DE, Wang R, Yu XH, Falagas ME. Fosfomycin: evaluation of the published evidence on the emergence of antimicrobial resistance in Gram-negative pathogens. J Antimicrob Chemother. 2012;67:255-68.

12. Raz R. Fosfomycin: an old-new antibiotic. Clin Microbiol Infect. 2012;18:4-7.

13. Flamm RK, Sader HS, Farrell DJ, Jones RN. Summary of ceftaroline activity against pathogens in the United States, 2010: report from the Assessing Worldwide Antimicrobial Resistance Evaluation 
(AWARE) surveillance program. Antimicrob Agents Chemother. 2012;56:2933-40.

14. Sader HS, Flamm RK, Streit JM, Farrell DJ, Jones RN. Ceftaroline activity against bacterial pathogens frequently isolated in US medical centers: results from five years of the AWARE surveillance program. Antimicrob Agents Chemother. 2015;59:2458-61.

15. Clinical and Laboratory Standards Institute. Performance standards for antimicrobial susceptibility testing; twenty-fourth informational supplement. CLSI document M100-S24. Wayne: Clinical and Laboratory Standards Institute; 2014.

16. Check-Points Health. Check-MDR CT101 user manual version 2.1. Wageningen: Check-Points Health; 2012.

17. Bogaerts P, Hujer AM, Naas T, et al. Multicenter evaluation of a new DNA microarray for rapid detection of clinically relevant bla genes from beta-lactam-resistant Gram-negative bacteria. Antimicrob Agents Chemother. 2011;55:4457-60.

18. Clinical and Laboratory Standards Institute. Methods for dilution antimicrobial susceptibility tests for bacteria that grow aerobically; approved standard, 9th edn. CLSI document M07-A9. Wayne: Clinical and Laboratory Standards Institute; 2012.

19. Hirsch EB, Raux BR, Zucchi PC, et al. Activity of fosfomycin and comparison of several susceptibility testing methods against contemporary urine isolates. Int J Antimicrob Agents. 2015;46:642-7.

20. Sanchez GV, Master RN, Karlowsky JA, Bordon JM. In vitro antimicrobial resistance of urinary Escherichia coli isolates among US outpatients from 2000 to 2010. Antimicrob Agents Chemother. 2012;56:2181-3.

21. Karlowsky JA, Denisuik AJ, Lagace-Wiens PR, et al. In vitro activity of fosfomycin against Escherichia coli isolated from patients with urinary tract infections in Canada as part of the CANWARD surveillance study. Antimicrob Agents Chemother. 2014;58:1252-6.

22. Hirsch EB, Zucchi PC, Chen A, et al. Susceptibility of multidrug-resistant Gram-negative urine isolates to oral antibiotics. Antimicrob Agents Chemother. 2016;60:3138-40.

23. Lai B, Zheng B, Li Y, Zhu S, Tong Z. In vitro susceptibility of Escherichia coli strains isolated from urine samples obtained in mainland China to fosfomycin trometamol and other antibiotics: a 9-year surveillance study (2004-2012). BMC Infect Dis. 2014;14:66.

24. Linsenmeyer K, Strymish J, Weir S, et al. Activity of fosfomycin against extended-spectrum-beta-lactamase-producing uropathogens in patients in the community and hospitalized patients. Antimicrob Agents Chemother. 2016;60:1134-6.

25. Mazzulli T, Skulnick M, Small G, et al. Susceptibility of community Gram-negative urinary tract isolates to mecillinam and other oral agents. Can J Infect Dis. 2001;12:289-92.

26. Sato N, Kawamura K, Nakane K, Wachino J, Arakawa Y. First detection of fosfomycin resistance gene fosA3 in CTX-M-producing Escherichia coli isolates from healthy individuals in Japan. Microb Drug Resist. 2013;19:477-82. 